Recibido: $18 / 02 / 2019$

Aceptado: 06/03/2019

\title{
Transformación pedagógica de planes educativos tradicionales a formación por competencias en Instituciones de Educación Superior colombianas
}

Pedagogical transformation of traditional educational plans to skills training in Colombian Higher Education Institutions

\author{
Mónica Cecilia Alzate De León \\ mcalzate@misena.edu.co \\ Lina María Otálvaro Rincón \\ lina09@gmail.com \\ Universidad Metropolitana de Educación, Ciencia y Tecnología \\ UMECIT. Panamá \\ Cira de Pelekais \\ cira.fernandez@floridaglobal.university \\ Florida Global University
}

\section{RESUMEN}

Este trabajo estuvo orientado en realizar un análisis de la transformación de planes educativos tradicionales a la formación centrada en competencias en Instituciones de Educación Superior en Colombia. Bajo un enfoque postpositivista, paradigma cualitativo, la metodología utilizada es la observación participativa, la población quedó conformada por 56 informantes clave, entre ellos docentes, estudiantes, directivos, administrativos y egresados procedentes de 18 instituciones de educación superior. Como técnica la observación, el instrumento un formulario tipo entrevista semi estructurada con 17 reactivos, de respuesta cerrada, el cual fue aplicado vía web y de manera presencial. Evidenciándose que la educación tradicional es predominante en las instituciones de educación superior en Colombia, tanto en lo administrativo como en lo académico. La planeación educativa está centrada en la administración de tiempos y recursos. Su gestión se encuentra desligada del sector productivo, por cuanto no existen convenios de cooperación que los relacione permanentemente.

Palabra clave: Modelo administrativo, Transformación pedagógica, Planes educativos, Competencias

\section{ABSTRACT \\ This work was oriented in carrying out an analysis of the transformation of traditional educational plans to the training focused on competencies in Higher Education Institutions in Colombia. Under a postpositivist approach, qualitative paradigm, the methodology used}


is participatory observation; the population was made up of 56 key informants, including teachers, students, managers, administrative and graduates from 18 higher education institutions. As an observation technique, the instrument is a semi-structured interview form with 17 reagents, with a closed response, which was applied via the web and in person. Evidence that traditional education is predominant in higher education institutions in Colombia, both administratively and academically. Educational planning is focused on the administration of time and resources. Its management is detached from the productive sector; since there are no cooperation agreements that permanently relate them.

Key word: Administrative model, Pedagogical transformation, Educational plans, Skills

\section{INTRODUCCIÓN}

La inserción laboral, es un elemento que debe ser tenido en cuenta en la educación, proporciona herramientas, conceptualizaciones y actividades desencadenadoras de desempeños efectivos a la toma de decisiones, conducen a la productividad de las empresas, objetivo de cada estudiante al finalizar su educación superior.

Los elementos articuladores de educación superior, son aquellos que generan integración del conocimiento, con el desempeño, el hacer, la actitud, la aptitud, el empresarismo, la idealidad de las cosas, creatividad, entre otros, que se espera según los retos de la educación, sean aplicados por los estudiantes al insertarse al mundo laboral.

De allí, que la educación a nivel superior busca crecimiento y transformación en el ser humano, en aspectos como los cambios en la mentalidad, aspiraciones personales, proyecto de vida, libre pensamiento, calidad de vida, reconocimiento, superación, por ello, los programas ofertados deben promoverlos a sus estudiantes.

El conocimiento es un arte de continua edificación, tan básico, como de alto nivel, así lo consagra la política de educación de un país, región o institución educativa, el saber se adquiere con el aprendizaje a lo largo de la vida, como práctica de vida, para enfrentar los desafíos de un mundo globalizado, de rápidos cambios en la información y en la tecnología. Poseer conocimientos aporta a la resolución de problemas cotidianos de vida, de la sociedad y situaciones laborales. El saber, proporciona seguridad, liderazgo, capacidad de decisión, evaluación, perspectiva, comprensión en el actuar y solución de temas para el ejercicio de la profesión.

Los académicos desde sus círculos universitarios estudian, analizan el mundo desde las necesidades del sector productivo y sus requerimientos de mano de obra cualificada, lo cual 


\section{Revista científica, arbitrada e indizada, bajo la modalidad electrónica.}

implica la revisión, replanteamiento de los programas de formación, la diversidad en la oferta de planes de pregrado, postgrado, diplomados y temas de extensión académica que cumplan con las expectativas de los empresarios en cuanto a condiciones; bajo este contexto, nace el planteamiento de implementar un currículo basado en competencias a nivel de la educación superior en Colombia.

De igual forma, la inserción laboral en la formación por competencias es el factor diferenciador, ya que tanto el estudiante como el empresario tienen información básica relacionada al logro del aprendiz en cuanto a su capacidad de realizar tareas, lo que la empresa espera de su parte, en cuanto al desempeño, calidad, productividad y eficiencia.

En consecuencia, la formación basada en competencias, permite aumentar la producción temprana del estudiante en su etapa productiva, ya que al tener claras sus capacidades de perfil de salida, la práctica laboral contribuye al perfeccionamiento y complementación de su profesionalidad hasta lograr la experiencia requerida. Un mundo globalizado requiere movilidad de los estudiantes, sean profesionales, tecnólogos o técnicos, para demostrar talentos, innovación y superación de dificultades.

Algunas universidades, no poseen la disposición institucional para cambiar su estilo educativo hacia la propuesta que involucra la formación de competencias, la cual, suscita a una reorientación en los procesos de planeación en la administración, gestión docente, implica capacitación permanente hacia la comunidad académica y la creación de unidades técnicas de apoyo pedagógico.

De igual forma, el rol del docente en la formación por competencias, está centrado en su experiencia práctica, académica, experimental, inscrita a un rol protagónico, emisor en el acto educativo, centrado en lo teórico, pero demostrado o referenciado; por otro lado, se encuentran los estudiantes tradicionales, con su papel receptor, pasivo, poco contundente, a lo que se requiere integrar disciplinas, saberes, haceres y teorías, bajo un trabajo coordinado de docentes, buscando evaluación de los aprendizajes teórico-prácticos, la medición personalizada del logro de cada aprendiz para la aplicación en el mundo real.

Considerando lo anterior, este trabajo tiene como propósito realizar un análisis de la transformación de planes educativos tradicionales a la formación centrada en competencias en Instituciones de Educación Superior en Colombia. 


\section{Revista científica, arbitrada e indizada, bajo la modalidad electrónica.}

\section{Situación problemática}

En las instituciones de educación superior colombianas, los procesos administrativos están regidos por reformas estatales dadas por el Ministerio de Educación Nacional, las cuales deben ser adoptadas y alineadas por toda entidad pública y privada que preste servicio educativo. Con respecto a lo planteado, hace más de dos décadas se viene cambiando el foco en la formación superior, desde programas exclusivamente teóricos a formatos que integren el desarrollo de competencias incorporadoras, genéricas, innovadoras, técnicas, transversales, laborales, en donde los desafíos se direccionen en la actualización de la administración educativa tales como: en modelos administrativos, planes educativos, metodologías de enseñanza - aprendizaje - evaluación, técnicas de desarrollo e innovación curricular.

En consecuencia, la educación tradicional cuestiona su calidad en la pertinencia de los aprendizajes y la aplicación de estos en el mundo laboral, adicional, su desactualización con respecto a las demandas cambiantes de un mundo globalizado. No obstante, la educación por competencias logra un aprendizaje más efectivo ya que se basa en métodos instructivos, componentes formativos socio- humanistas que favorecen el desarrollo de capacidades, equilibrio personal, relación interpersonal, inserción social, desarrollo cognitivo, entre otros. En este sentido, las instituciones de educación superior colombianas han de ser críticas, ya que el egresado no solamente deberá contar con formación científica sino también técnica, para poder dar respuesta a las necesidades del sector productivo en cuanto a su capacidad de solucionar problemáticas ante las dinámicas actuales.

\section{Conceptos definidores y sensibilizadores}

La transformación de un modelo educativo tradicional a un modelo por competencias, es un gran desafío institucional, requiere centralizar esfuerzos, comprometidos con el progreso el bienestar regional y del país, en permanente diálogo e interacción con el entorno social, cultural y económico, de esta manera se pretende que el modelo educativo por competencias, genera un fuerte cambio a las instituciones de educación superior, tanto en su planificación estratégica, como en su estructura organizacional.

Al respecto Pizarro (2014), menciona que el modelo de la educación por competencias implica no solo un desarrollo por encima de la visión de los estudiantes como futuros 


\section{Revista científica, arbitrada e indizada, bajo la modalidad electrónica.}

profesionales, sino perfeccionar la manera como éste se desenvuelve en el ámbito empresarial, promoviendo las capacidades analíticas, reflexivas, de medir, evaluar las externalidades positivas o negativas de las decisiones adoptadas.

Un modelo de educación por competencias debe generar para las instituciones de educación superior la oportunidad de mejorar la calidad en sus programas de formación, enfoque que ha sido promovido desde la Unión Europea, a partir de la Declaración de Bolonia en el año 1999 y por el Proyecto Tuning para la formación universitaria, mostrando resultados importantes y diferenciadores para los egresados, pero que exigen una transformación institucional desde su gestión administrativa.

Según Angulo y Rendón (2011), este proceso en países como España ha dado origen a fuertes cambios en la planificación estratégica de las universidades y por consecuencia en su estructura organizativa, en donde las metas antes propuestas y los indicadores para esta, también han sufrido fuertes cambios.

Actualmente, se asume que la tarea fundamental en el rediseño de las instituciones educativas es revisar la disociación existente entre lo pedagógico y lo organizacional, las transformaciones educativas radican en una gestión integrada institucional: calidad, equidad, pertinencia del currículo y profesionalización de las acciones educacionales.

Por lo tanto, el enfoque para el desarrollo de competencias en la administración educativa, se basa en propuestas que pueden ser de carácter humanista, cognitiva, sistémica, flexible o permanente, se hace presente en el proceso formativo, buscando el desarrollo Integral del estudiante, a partir de la articulación entre lo tecnológico, administrativo, social y laboral, como medio para el desarrollo de procesos cognitivos, procedimentales, valorativos, actitudinales , permitiendo el actuar crítico y creativo en todos los aspectos de la gestión administrativa institucional. (García Retana, 2011).

La administración en las instituciones de educación superior, tiene orientación, contenidos diversos, es vista como una acción de apoyo, puesto que fija los programas, escoge a los profesores, determina la organización del lugar, define las instalaciones, establece la composición del grupo de docentes y administrativos, también dispone, reglamenta todo lo concerniente a la estructura de la institución a las condiciones de su desenvolvimiento. (Vargas, 2011). 
Ahora bien, el resultado de la administración educativa tradicional ha sido la existencia de organizaciones cuyas acciones en muchos casos no se interconectan, es centralizada, deja poco a la iniciativa y a la mejora inmediata de la institución de educación.

En consecuencia, administrar un sistema educativo es representar la institución que dirige o la red escolar que coordina, a fin de satisfacer las necesidades de los diferentes actores internos o vinculados a la institución y así contribuir a cubrir la demanda cuantitativa y cualitativa de educación. Todo directivo al gerenciar una institución educativa aplica, de manera continua, en conjunto con los demás actores, el ciclo es planificar-ejecutar-revisaractuar. (OECD, 2016).

\section{Planificación y coordinación}

En la planeación y coordinación de instituciones de educación, un director académico tendrá que liderar acciones encaminadas a planear, coordinar actividades de aprobación de políticas académicas, métodos, programas de enseñanza, investigación o difusión académica; también se encargará de velar por la efectividad de los procesos de acreditación de alta calidad, el diseño de nuevos programas o su actualización, desarrollo curricular y seguimiento a los objetivos estratégicos de la universidad.

La coordinación de responsabilidades del director académico incluye en su papel el liderazgo en el servicio, ser confiable, desempeñarse éticamente, promover la disciplina académica en los estudiantes y egresados. (Montemayor, 2011)

Los directores académicos dentro de su planeación y coordinación institucional responden a la globalidad institucional: profesores, administración central, estudiantes, comunidad, sociedad, al gobierno del país, ya que se responsabiliza de los cambios institucionales, relacionados con el organigrama y su aporte a las diferentes áreas.

Para los administradores, las áreas de trabajo tienen una evolución a la par con el crecimiento de la institución, por ello un coordinador planea, controla y está dispuesto a solucionar las diferentes situaciones que se presenten; esta figura también es visionaria, promociona las necesidades, puntos de conexión entre metas estratégicas, objetivos institucionales y el proyecto educativo institucional. (Montemayor, 2011)

En la planificación, el directivo con su equipo decide qué hacer y cómo hacerla, su fin es convertir la institución de educación en un centro de excelencia pedagógica, alineado a su 
proyecto educativo institucional, orientador de los procesos de enseñanza en el aula, a partir de un diagnóstico de su realidad, la fijación de objetivos a lograr, los cursos de acción a seguir y los recursos requeridos. (Graffe, 2002)

La planificación en la gestión administrativa tradicional en las instituciones de educación, se da como un proceso sistemático orientado al fortalecimiento de las instituciones educativas y a sus proyectos, con el fin de enriquecer los procesos pedagógicos, directivos, administrativos. En la planificación se permite conservar cierta autonomía institucional para así responder de una manera más acorde, a las necesidades educativas locales, regionales. (Nacional, 2018)

\section{Organización}

Algunas de las áreas en las cuales los directores académicos deben impactar son entre otras: La búsqueda del conocimiento; el compromiso y coherencia del aprendizaje para la vida; ajustarse a los cambios permanentes, apoyar la evolución de la institución y formar adecuadamente en búsqueda de competencias.

En otras palabras, el reto más importante del director académico es liderar, en condiciones variables como el contexto, la sociedad, el desarrollo económico, la productividad, también guiar a la comunidad educativa en función del cumplimiento de políticas administrativas y estatales.

La organización se asocia con la planeación o la distribución de recursos para poder realizar la formación con lo requerido y buscando que el estudiante disponga de las condiciones adecuadas que favorezcan su proceso educativo.

\section{Dirección}

El desarrollo profesional de los directivos educativos se vincula a los procesos de mejora organizativa, así mismo se ve como un requisito necesario que posibilita el cumplimiento de metas y objetivos en institución educativa, también, es una herramienta propia que actúa al servicio de la organización.

En este sentido, los modelos de formación tradicional o lineal han quedado obsoletos dando origen a estrategias y modelos de formación como el basado en competencias o 'gestiónacción’ que combina aspectos profesionales y la mejora técnica. (Díaz Villa, 2002). 
Un director, es el responsable de planificar, dirigir, supervisar, ejecutar las actividades académicas, administrativas y financieras de una facultad o institución de educación superior; según Ramírez (2013), dentro de sus principales funciones se puede referenciar lo siguiente:

- Identificar, formular o depurar la oferta académica de una institución, teniendo en cuenta su pertinencia, fortalezas, oportunidades del medio o áreas del conocimiento en las cuales se requiera impartir formación según la demanda del sector productivo.

- Diseñar mecanismos para el aseguramiento de la calidad, pertinencia de los programas académicos, currículos, los sistemas de enseñanza - aprendizaje - evaluación y personal docente.

- Implementar políticas, relacionadas con los lineamientos de educación superior, normas para la gestión, el aseguramiento de la calidad, nuevas oportunidades para la obtención de acreditaciones y certificaciones nacionales e internacionales que demuestren la excelencia de los programas académicos.

- $\quad$ Elaborar informes y reportes de gestión que incluyan los indicadores del proyecto educativo institucional para la toma de decisiones.

Así mismo, la dirección, se asocia con el liderazgo, la motivación y la creación de un clima organizacional por la parte directiva, que integre las potencialidades en los diferentes sujetos, a partir del compromiso de todos con el proyecto educativo para mejorar la docencia y la administración en la institución de educación.(UNESCO, 2017)

De hecho, la dirección en la gestión administrativa en educación tradicional tiene que ver con gobernabilidad, balances e integraciones necesarias entre lo técnico, político en educación; mediante esta posición estratégica de las prácticas de dirección organizaciones educativas puede hablarse de gestión. También tiene que ver con los problemas los cuales refieren a la resolución de conflictos planteados entre previsto y contingente, entre lo formalizado y rutinario. (IIPE-UNESCO, 2017)

Dado que en la gestión administrativa tradicional la dirección es poco rigurosa, se podría decir dispone sólo lo indispensable. Las relaciones del gobierno central con las instituciones educativas con otras instituciones y entidades que se ocupan de la educación, se desarrollan en un cierto plano de igualdad. Están basadas principalmente en la cooperación y la consulta. (Hernandez, 2014) 
No obstante, han aparecido unas instituciones de educación superior, que reclaman una dirección y gestión en el seno del mismo, es decir, una administración educativa interna con la que habrá de articular su actuación. (Lázaro, 2012)

\section{Control}

Dirigir, organizar, formular políticas, adoptar planes, programas y proyectos requieren de un control permanente para así contribuir con el desarrollo social, económico, educativo, tecnológico del país, en cumplimiento de la misión, visión y objetivos institucionales de la Educación Superior. (SENA, 2014a)

El control permite asegurar la ejecución de la programación de acuerdo con el esquema de responsabilidades, distribución del trabajo que se diseñó, para lograr los objetivos, metas asignados a los diferentes actores o unidades de la institución; e introducir ajustes a la programación y a la asignación de recursos (Zapata, 2015).

Ahora bien, el control y seguimiento de la gestión no se puede realizar sin datos e información. Dato es el resultado de una medición cuantitativa o cualitativa, obtenida para dirigir tomando decisiones racionales. Pero si los datos son analizados permiten tener una interpretación del desempeño de los diversos procesos, programas y proyectos; actividad de mayor valor agregado que permite identificar las desviaciones o variaciones a las ejecuciones previstas para establecer las causas de las diferencias detectadas. (Graffe, 2002).

Hay que mencionar, además el control en las instituciones de educación superior, es una herramienta que permite obtener un apropiado manejo de la institución, permitiendo garantizar las metas con respecto a la formación por competencias desde un punto de vista operativo y normativo. (Espinoza Cruz, 2009)

Bajo esta línea Ramos (2009) señala que el control interno pretende:

- Aumentar la probabilidad de realización de los objetivos.

- Reducir o prevenir los riesgos.

- Disminuir las sorpresas en los procesos.

- Asegurar la credibilidad de la información.

- Cumplir las leyes y normas.

- Rendir cuentas. 
Por otro lado, dentro del marco constitucional se definen algunos organismos de control administrativo y disciplinario para las instituciones de educación superior colombianas tales como:

Procuraduría General de la Nación: Esta institución ejerce vigilancia en cuanto a la conducta de los funcionarios que trabajan para el estado, en cuanto a investigaciones de actos indebidos, sanciones y seguimiento de la ley según el artículo 277 de la Constitución Política de Colombia de 1991. (PROCURADURIA, 2018) (ITSA, 2018).

Contraloría General de la Nación: dentro de su institucionalidad se derivan funciones relacionadas con el control de la calidad, eficiencia del control fiscal, organismos públicos, jurisdicción, es decir evalúa la calidad, el nivel de confianza, eficiencia del Sistema de Control Interno de las entidades que conforman el estado, según la Ley 42 de 1993 y Ley 87 de 1993. (CONTRALORIA, 2018), (ITSA, 2018)

Departamento Administrativo de la Función Pública: este organismo encargado de dar directrices administrativas y de políticas, para el mejoramiento de las Instituciones estatales y el desarrollo de sus funcionarios (CONTRALORIA, 2018),(ITSA, 2018).

Comisión Nacional del Servicio Civil: La CNSC está encargada de velar por la administración y vigilancia de las carreras de servidores públicos dentro de las funciones estatales de concurso y meritocracia. (COMISION NACIONAL, 2018),(ITSA, 2018)

Ministerio de Educación Nacional: El MEN es el ministerio gubernamental que se encarga de establecer los lineamientos, vigilar y controlar la Educación en Colombia.

\section{Coordinación}

Con respecto a la coordinación, su finalidad en las instituciones de educación superior tradicionales es la de promover una enseñanza de calidad a partir de una mayor coherencia entre los procesos de intervención formativa. Dentro de los objetivos de la coordinación en la educación tradicional se encuentran:

- Proporcionar un conocimiento compartido sobre la realidad y las necesidades educativas y personales de los estudiantes.

- Promover acuerdos sobre modelos, sistemas y estrategias de intervención educativa contextualizada y coordinada. 
- Impulsar la coordinación de los proyectos curriculares en las materias comunes que formen parte del currículo obligatorio. (Martínez \& Echeverría, 2009).

Otro rasgo de la coordinación es asegurar que haya un proyecto de formación coherente para los estudiantes, estableciendo mecanismos de seguimiento y mejora permanente. Los contenidos propios de la coordinación pueden estar relacionados con temas de gestión institucional, con los aspectos curriculares, con temáticas extracurriculares o con actuaciones vinculadas al medio natural y social. (Ministerio de educación, 2011) .

En este sentido, se asume que la tarea fundamental en el rediseño de las instituciones educativas es revisar la disociación existente entre lo pedagógico y lo organizacional, las transformaciones educativas radican en una gestión integrada institucional: calidad, equidad, pertinencia del currículo y profesionalización de las acciones educacionales. (García Retana, 2011).

El enfoque para el desarrollo de competencias en la administración educativa, se basa en propuestas que pueden ser de carácter humanista, cognitiva, sistémica, flexible o permanente, se hace presente en el proceso formativo, buscando el desarrollo Integral del estudiante, a partir de la articulación entre lo tecnológico, administrativo, social y laboral, como medio para el desarrollo de procesos cognitivos, procedimentales, valorativos, actitudinales , permitiendo el actuar crítico y creativo en todos los aspectos de la gestión administrativa institucional. (García Retana, 2011).

\section{Concepto de competencia}

Así mismo, el concepto de competencia se estructura con base en tres aspectos fundamentales coherentes con los principios de integralidad, equidad social, trabajo productivo y la formación permanente propios de la Formación Profesional Integral. (Paredes \& Inciarte, 2014).

\section{Desarrollo Integral}

En alusión al fortalecimiento de las dimensiones humanas del estudiante como persona, ciudadano y trabajador en formación (aprender a ser), gestor de su propio desarrollo, con capacidades para interactuar y aportar a la construcción de sociedad sobre la base de sólidos valores éticos. 


\section{Equidad social}

Hace relación con el proceso de construcción de conocimiento y el carácter social del mismo (aprender a aprender), lo cual exige del estudiante una actitud de empoderamiento de su proceso de aprendizaje, a partir del ejercicio de autonomía y de autogestión como oportunidades que le brindan las estrategias didácticas de carácter activo para el desarrollo del proceso de formación. (SENA, 2014a).

\section{Trabajo productivo}

Para el diseño del modelo, se debe pensar en el trabajo productivo como escenario propicio para los desempeños, como condición ineludible del desarrollo de competencia, cuyo efecto se refleja en la transformación intencional del entorno físico y social, para lo cual requiere del desarrollo de las habilidades y destrezas (aprender a hacer) propias de la dimensión biofísica, con el fin de desarrollar la competencia laboral en el estudiante, teniendo en cuenta además, los criterios establecidos por el sector productivo, según el objeto de formación. (Paredes \& Inciarte, 2014).

\section{Enfoque pedagógico del modelo tradicional en la educación y la formación por competencias.}

Para revisar adecuadamente el enfoque pedagógico es necesario realizar un cuadro comparativo entre las variables asociadas:

\section{Cuadro 1.}

Variables asociadas a los diferentes enfoques

\begin{tabular}{|c|c|c|c|}
\hline & & TRADICIONAL & COMPETENCIAS \\
\hline $\begin{array}{l}\text { METODOLOGIA } \\
\text { DIDÁCTICA } \\
\text { DESARROLLO }\end{array}$ & $\mathbf{Y}$ & $\begin{array}{l}\text { Conductista } \\
\text { Cognoscitivismo } \\
\text { Formación periódica semestral } \\
\text { o anual } \\
\text { Mide resultados }\end{array}$ & $\begin{array}{l}\text { Constructivismo } \\
\text { Paradigma sociocultural } \\
\text { Corriente humanista } \\
\text { Proceso continuo }\end{array}$ \\
\hline EVALUACION & & $\begin{array}{l}\text { Énfasis en resultados } \\
\text { Medición y sanción } \\
\text { Instrumentos estadísticos } \\
\text { Estandarizados } \\
\text { Evaluación al final } \\
\text { Poca diversidad } \\
\text { Evaluación escrita, Preguntas } \\
\text { abiertas, respuesta fija o elección } \\
\text { múltiple. }\end{array}$ & $\begin{array}{l}\text { Enfoque a competencias y } \\
\text { desempeños individuales } \\
\text { Evaluación durante todo el } \\
\text { proceso formativo } \\
\text { Diversidad en instrumentos de } \\
\text { evaluación } \\
\text { Permite La toma de decisiones y } \\
\text { la mejora continua } \\
\text { Variedad en la evaluación mapa, }\end{array}$ \\
\hline
\end{tabular}




\begin{tabular}{|c|c|c|}
\hline & TRADICIONAL & COMPETENCIAS \\
\hline & & ensayos, debates, etc. \\
\hline RECURSOS & $\begin{array}{l}\text { Locativos, documentales, } \\
\text { magistrales y tradicionales }\end{array}$ & $\begin{array}{l}\text { Multimediales } \\
\text { Teórico-prácticos } \\
\text { Vivenciales } \\
\text { Laboratorios } \\
\end{array}$ \\
\hline PROPÓSITOS Y METAS & $\begin{array}{l}\text { Evaluación del aprendizaje por } \\
\text { contenidos } \\
\text { Medir resultados y sancionar al } \\
\text { final del proceso }\end{array}$ & $\begin{array}{l}\text { Evaluación de la enseñanza y el } \\
\text { aprendizaje con el desarrollo de } \\
\text { competencias } \\
\text { Retroalimentar } \\
\text { Asegura el mejoramiento }\end{array}$ \\
\hline $\begin{array}{l}\text { CONTENIDOS } \\
\text { CURRICULAR }\end{array}$ & $\begin{array}{l}\text { Materias } \\
\text { Asignaturas } \\
\text { Pre-requisitos } \\
\text { Conocimientos } \\
\text { Cursos }\end{array}$ & $\begin{array}{l}\text { Módulos } \\
\text { Actividades } \\
\text { Objetivos } \\
\text { Resultados } \\
\text { Saber - hacer - ser }\end{array}$ \\
\hline ROL DOCENTE & $\begin{array}{l}\text { Magistralidad } \\
\text { Normativo } \\
\text { Comparativo } \\
\text { Activo en el discurso }\end{array}$ & $\begin{array}{l}\text { Auto evaluativo y expositivo } \\
\text { Incita al trabajo colaborativo } \\
\text { Retroalimenta y apoya al } \\
\text { estudiante } \\
\begin{array}{l}\text { Busca el mejoramiento del } \\
\text { estudiante }\end{array}\end{array}$ \\
\hline ROL ESTUDIANTE & $\begin{array}{l}\text { Receptor } \\
\text { Pasivo } \\
\text { Solitario } \\
\text { Inseguro }\end{array}$ & $\begin{array}{l}\text { Emisor y multiplicador } \\
\text { Activo } \\
\text { Trabaja en equipo } \\
\text { Emprendedor }\end{array}$ \\
\hline
\end{tabular}

Fuente: Elaboración propia (2018).

\section{Modelo de educación por competencias}

El término competencia nace en el sector laboral, centrado al sector técnico, con la premisa del saber, saber hacer y saber ser, además tomando en cuenta los pasos a seguir para obtener un resultado en particular.

Según Le Bortef (2001, pág. 122), la competencia es una construcción, a partir de una combinación de recursos como los conocimientos, el saber hacer, las cualidades o aptitudes, los recursos del ambiente como relaciones con terceros, documentos e informaciones obtenidas de investigaciones además las conclusiones de otros, que son movilizados para lograr un desempeño. Las competencias se adquieren de manera por lo general circunscrita, simple, mecánica, rutinaria, repetitiva, tangibles, vinculadas al ámbito de la capacitación y desempeño en el mundo laboral.

A partir de esto, es posible inferir que las competencias son las habilidades para realizar trabajos de manera práctica, se extienden al saber conocer, saber ser, saber estar, las cuales combinadas, activadas y puestas en acción permiten movilizar en contextos específicos 


\section{Revista científica, arbitrada e indizada, bajo la modalidad electrónica.}

acciones para resolver una familia de situaciones o problemas por los estudiantes, demandan capacidades que van más allá de las procedimentales, cognitivas, capacidades del día a día con mayor relevancia como las actitudinales, intrapersonales e interpersonales. (Rodicio García \& Iglesias Cortizas, 2011).

Por su parte, Escudero (2013), expone una de las razones más discutidas entre los especialistas en competencias es la multidimensionalidad del término, en él interactúan diversos elementos, dependiendo de las disciplinas o contextos desde donde se aborde.

De acuerdo con Suárez (2007), esta gran discusión conceptual se debe, a la dificultad de determinar la naturaleza de las competencias y definir cuáles son las condiciones idóneas de formación para su desarrollo, asimismo, Iglesias (2011) subraya, ya sea en el contexto emocional o profesional en el que se realicen interpretaciones, sus múltiples significados se encuentran vinculados a la capacitación en conocimientos, actitudes y habilidades de una persona, como resultado de su preparación adquirida.

Estos antecedentes dejan ver la presencia de elementos comunes en la discusión respecto al término competencia, en donde uno de los más aceptados por los investigadores especialistas es el de Armengol et al., (2011). El cual describe que las competencias son consideradas como un conjunto de conocimientos, habilidades, actitudes que se complementan entre sí, atendiendo a los atributos personales de cada individuo, a las experiencias personales, profesionales, capacitándole para actuar con cierto nivel de calidad y eficacia en un entorno determinado.

Para García (2011), a pesar de no existir una teoría unificadora de las competencias, dicha integración supone operaciones mentales complejas en las que el individuo moviliza sus características personales, formativas y vivenciales, a fin de desempeñarse eficazmente y ser consciente de cada una de sus acciones.

Sin embargo, se contempla que las competencias son a veces entendidas como una mezcla entre conocimientos teórico- prácticos permitiendo un aprendizaje carente de sentido, por ello debe mirarse a los individuos constructores del aprendizaje de sus competencias movilizando recursos tales: actitudes, aptitudes, saber hacer, cualidades personales, experiencias, en donde hacen, se destacan las aptitudes de trabajar en equipo, bajo presión, orientado a resultados; capacidad de dialogar, escuchar al otro, expresarse, comunicarse por escrito, en forma gráfica, oral, además de reflexionar sobre su hacer y el de los demás. 
En consecuencia, la educación basada en competencias lejos de ser una educación fragmentada, de corte conductual, tiene ventajas que inciden significativamente en diferentes áreas del proceso educativo, abriendo perspectivas más dinámicas, integrales y críticas. (López Ortega, 2008).

En consecuencia, se requiere de una orientación educativa que dé respuesta una actualidad en las industrias, el concepto de competencia, tal y como se entiende en la educación, resulta de las nuevas teorías de cognición (inteligencias múltiples) y básicamente significa saberes de ejecución.(Álvarez et al., 2015).

Por otra parte, en 1998 la UNESCO expresa en la Conferencia Mundial sobre la Educación Superior, que es necesario propiciar el aprendizaje permanente, la construcción de competencias adecuadas para contribuir al desarrollo cultural, social y económico de la sociedad. Asimismo, ha señalado que las principales tareas de la educación superior han estado y seguirán estando ligadas a cuatro de sus funciones principales:

- Construir una generación con nuevos conocimientos (las funciones de la investigación).

- Obtener el entrenamiento de personas altamente calificadas (la función de la educación).

- Proporcionar servicios a la sociedad (la función social).

- La función ética, que implica la crítica social.

\section{Tipos de competencias}

Son múltiples las clasificaciones de competencias que se conocen, cada una de ellas están sujetas al contexto en donde se implementan y los propósitos formativos que persigue cada institución educativa, se enuncian algunas.

\section{Competencias Específicas}

Las competencias específicas Identifican el desempeño en el contexto de una función productiva, la cual requiere para su ejecución de la aplicación de conocimientos técnico tecnológicos que den respuesta pertinente a los requerimientos en forma de estándares, establece el sector productivo, los que determinan las condiciones técnicas y de calidad requeridas en un desempeño satisfactorio.(Paredes \& Inciarte, 2014). 


\section{Competencias Básicas o clave}

Es conveniente subrayar que las competencias básicas o clave son un conjunto dinámico de capacidades para el desempeño en el mundo de la vida que facilitan a la persona su inserción y permanencia en el mundo del trabajo. Le permiten comprender, argumentar, resolver problemas tecnológicos, sociales, ambientales. Se desarrollan de modo permanente en el proceso de formación profesional y su nivel o grado de complejidad está asociado al nivel de exigencia requerido en el ámbito social o laboral (Paredes \& Inciarte, 2014).

Algunas de las principales características de este tipo de competencias son:

- Son indispensables para el Desarrollo Humano Integral en las dimensiones personal y social, fundamentan el fortalecimiento de comportamientos éticos con el dominio de procesos de comunicación asertiva necesarios para la interacción social, convivencia, iniciativa, creatividad en la solución de problemas sociales y productivos con base a la aplicación de conocimiento. (SENA, 2014a).

- Son de carácter abierto; hacen parte constitutiva de la necesidad de aprendizaje permanente, a la vez que trasciende toda actividad humana, esenciales para el desarrollo de cualquier otro tipo de competencias, por lo cual adquieren el carácter de ser generativas y transversales. (SENA, 2014a).

\section{Competencias Transversales}

Este tipo de competencias agrupan todas aquellas competencias necesarias para complementar los desempeños sociales y/o laborales, de carácter transversal, no están enmarcadas en ninguna disciplina específica, se caracterizan por su alto nivel de transferibilidad y flexibilización puesto que son condición necesaria para el desempeño del Aprendiz en los diferentes niveles ocupacionales. Su carácter transversal implica la condición de interdisciplinariedad en su desarrollo.(Ministerio de educacion nacional, 2016).

\section{Competencias personales}

Son aquellas que permiten la realización exitosa de las metas personales propuestas a lo largo de su vida como son: actuar con responsabilidad, espíritu de superación y aceptación al cambio, profesionalizarse, tener un buen empleo, entre otras. 


\section{Competencias centrales o nucleares}

Buscan el desarrollo humano para cada estudiante y algunos ejemplos son la comunicación, trabajo en equipo, responsabilidad, creatividad, orientación al cliente, compromisos con el aprendizaje continuo, entre otras. (Solar, 2005).

\section{Competencias de gestión}

Las competencias de gestión están enfocadas en el liderazgo, visión, construir confianzas, gerenciar el desempeño, capacidad de juicio y de toma de decisiones. (Bolívar, 2011).

\section{Competencias académicas}

Estas competencias están relacionadas con el tipo de didáctica, manejo de los componentes personales y no personales del proceso enseñanza-aprendizaje. También se relaciona con el tratamiento sistémico de las categorías, objetivos, contenido, método, medios, forma de enseñanza y la evaluación. (Marrero Sánchez \& Lasso, 2017).

\section{Competencias organizativas}

Estas competencias se enfocan en el dominio de todo lo relacionado con la planificación, organización, ejecución y control de las acciones pedagógicas, didácticas involucradas en la formación del que aprende. (Marrero Sánchez \& Lasso, 2017).

\section{Competencias genéricas}

Las competencias genéricas son aquellas que buscan en el estudiante el desarrollo de capacidades que le permitan hacer los procesos con calidad, se encuentran los ejes de: capacidad de aprender, capacidad de resolver problemas, capacidad de análisis y síntesis, capacidad de adaptarse a situaciones nuevas, capacidad para trabajar de forma autónomo, trabajo en equipo, habilidades para diseño y gestión de proyectos. (Marrero Sánchez \& Lasso, 2017).

\section{Competencias Marco}

Son los grandes propósitos de la educación, las metas a lograr en la formación, reflejan los aprendizajes de contenidos declarativos, procedimentales, actitudinales ligados a 
realizaciones o desempeños que los estudiantes deben manifestar, utilizando de manera pertinente, flexible en situaciones nuevas y desconocidas, al egresar de cada nivel; finalmente se toman en cuenta tanto los saberes socioculturales de los Pueblos del país como los saberes universales. (Ministerio de Educacion Guatemala., 2018).

\section{Competencias de Eje}

Señalan los aprendizajes que enlazan el currículum con los problemas, expectativas y necesidades sociales. Establecen una relación entre las actividades escolares y las dimensiones de la vida en comunidad. Dicha relación contribuye a definir la pertinencia de los aprendizajes. (Corado Fuentes, 2017).

\section{Competencias de Área}

Integran las capacidades, habilidades, destrezas y actitudes que los estudiantes deben lograr en las distintas áreas de la ciencia, las artes, la tecnología. Por ejemplo, el área de productividad - desarrollo. (Corado Fuentes, 2017).

\section{Competencias sub-área}

Son aquellas competencias que comprenden las capacidades, habilidades, destrezas y actitudes que los estudiantes deben lograr en las diferentes áreas del conocimiento durante su formación profesional. (Corado Fuentes, 2017).

\section{Competencias de grado}

Son aquellas competencias las cuales compilan relaciones o desempeños en el diario que hacer docente dentro y fuera del aula; implican un aprendizaje significativo en los estudiantes. (Corado Fuentes, 2017).

\section{Características de las competencias}

Las principales características de las competencias se describen a continuación:

\section{Carácter teórico-práctico}

Requieren saberes técnicos y académicos en relación con la acción en cierto lugar de trabajo, en un contexto determinado. 
Como señala Perrenoud (2004), es en su funcionamiento cuando toman sentido, en tanto que se relacionan con un grupo de problemas y tareas, llevando a movilizar, clasificar los recursos cognitivos (conocimientos, técnicas, habilidades...). Ejecutar una competencia implica tanto el desarrollo de operaciones mentales como la realización de acciones.

\section{Carácter aplicativo}

Esta característica permite un reconocimiento estructural entre los problemas o las situaciones presentadas durante la formación profesional, adicional a esto se requiere un repertorio de soluciones para situaciones variadas, finalmente incorpora la voluntad y capacidad para caracterizar las circunstancias a fin de convertirlas en oportunidades de trasferencia. (Cano, 2015).

\section{Carácter contextualizado}

Esta característica, permite que una competencia tome diferentes sentidos según la situación pedagógica, tratando que todo conocimiento adquirido sea aplicado a un proceso heterogéneo o múltiple, por lo anterior, el concepto de competencia se aplica a la reflexión, organización, seleccionan e integración, resolviendo un problema. (Cano, 2015).

\section{Carácter reconstructivo}

En esta característica, las competencias se crean, actualizan constantemente en la práctica profesional además de la aplicación real de funciones laborales, mas no inician en la formación de pregrado. Adicional a lo anterior, adquieren validez, sentido por su relación directa con la innovación permanente, investigación y creatividad. (Cano, 2015).

\section{Carácter combinatorio}

El carácter combinatorio involucra los conocimientos, los procedimientos, las actitudes, así como las capacidades de cada individuo, estas a su vez se complementan y combinan entre sí, para ser, en definitiva, competente en el saber, hacer y ser. (Cano, 2015).

\section{Carácter interactivo}

Dentro del modelo administrativo se debe implementar el carácter interactivo ya que este 
permite la adquisición, el desarrollo de competencias no solo individuales, sino también con la comunidad, con el contexto. El desarrollo de competencias no será individual, estas se mejoran al trabajar con otros, puesto que la búsqueda de soluciones y respuestas de un referente específico, se da en muchos casos en forma conjunta en el día a día. (Cano, 2015).

\section{MATERIALES Y MÉTODOS}

Esta investigación corresponde a un enfoque postpositivista de tipo cualitativo. En este sentido, los procedimientos de investigación desde un enfoque paradigmático cualitativo son flexibles, orientados a la exploración, el descubrimiento, permitiendo que durante el transcurso sea posible añadir, cambiar tipos $\mathrm{y}$ fuentes de datos en procura de un entendimiento más profundo de la realidad estudiada. Los métodos cualitativos de investigación han permitido durante los últimos años consolidarse como una estrategia asertiva además de exitosa en el sistema de educación superior para vincular a la universidad con el estudio de los fenómenos sociales desde diversos abordajes disciplinarios. (Leyton \& Herrera, 2012).

En el tema de la formación por competencias con el uso de métodos y herramientas, se ha permitido que los estudiantes reconstruyan la realidad a partir de nuevas interpretaciones sobre diversos fenómenos sociales que muchas veces son ajenos a su contexto de origen, como la pobreza, la inequidad, la violencia, el desempleo, ya sea en el desarrollo de sus programas de formación o a través de su acercamiento al mundo de la investigación desde el paradigma fenomenológico, fomentando su compromiso y sensibilidad social. (Díaz Barriga, 2006).

Considerar la formación por competencias como una investigación permite una experiencia para los autores que los obliga a definir un modelo integrador entre la posición de los estudiantes, docentes y administradores como insumos de alta importancia, el proceso implementado en su formación y una sucesión de productos logrados en la forma como cada uno de ellos presentó un avance en la toma de decisiones, comportamiento, madurez, compromiso, más allá de la preocupación sobre la calificación del curso. (Asún Inostroza et al., 2013).

Tomando en cuenta el enfoque paradigmático para el desarrollo de esta investigación, se identifica la formación por competencias a nivel de la educación superior como una 
realidad que en los últimos años tanto en el ámbito nacional como internacional se ha mantenido, la discusión de cómo debe ser el proceso de enseñanza aprendizaje, las competencias adquiridas en relación con su entorno, las capacidades que los egresados deben poseer al terminar sus estudios según las necesidades del mundo productivo y la realidad social. (Cabra Torres, 2008).

Desde el enfoque paradigmático se busca revisar si los resultados en el desempeño, implica modificar el modelo curricular, cuyo método de enseñanza-aprendizaje-evaluación, sea una experiencia práctica en donde se vinculen los conocimientos adquiridos en la formación. (Stabback, 2016).

Finalmente, la formación tradicional y la basada en competencias tienen diferencias donde se pueden mencionar temas tales como: la pertinencia del egresado en demanda, oferta laboral; la pertinencia en la calidad educativa; perfiles profesionales, académicos de los docentes además del cuerpo administrativo de la institución.

Estos aspectos permiten confirmar la elección de la tradición cualitativa en este trabajo, ya que se involucra todos los actores de la comunidad educativa en sus distintas realidades tales como es la educación superior en Colombia, la formación tradicional o la formación por competencias; aspectos importantes y relevantes en este contexto de estudio. (Muro Xiomara, \& Serrón Sergio, 2007).

Para este enfoque paradigmático cualitativo, se seleccionaron cincuenta y seis (56) personas, las actuaciones se desarrollan en el contexto administrativo y pedagógico, los planes educativos en las Instituciones de Educación Superior (IES) colombianas.

Los criterios de selección de_los informantes clave fueron:

- Muestreo por conveniencia: por el interés de obtener información significativa se solicitó a personas con diferentes cargos directivos en las IES, que diligenciaron la encuesta para poder evidenciar aspectos que son realizados desde este rol de directivos en las instituciones.

- Muestreo por voluntarios: Se solicitó a personas pertenecientes a diferentes instituciones de educación superior de carácter público o privado, en cuyo caso tuvieran los roles propuestos dentro de este trabajo, tales como docentes, estudiantes, egresados, realizar la encuesta y en algunos casos se les acompañó en su diligenciamiento. 


\section{Revista científica, arbitrada e indizada, bajo la modalidad electrónica.}

- $\quad$ Reflexividad: A cada una de las personas encuestadas se les contó por qué se realizaba la encuesta y se les invitó a realizar una reflexión sobre su rol en la institución de educación superior.

- Coherencia: Después de respondidas las encuestas, los resultados fueron revisados y en el momento de encontrar datos incoherentes se les solicita aclararlos con el fin de asegurar que el trabajo realizado sea válido.

- Autenticidad: Para esto se solicitó solo a personas conocidas que respondieron esta encuesta buscando la mayor sinceridad, de este modo obtener información real y confiable.

Las técnicas cualitativas aplicadas fueron: La observación directa, vivencial y de indagación, su aplicación permitió identificar por medio del contacto directo, el quehacer formativo de entidades de educación superior, en componentes como modelo administrativo, la evaluación, la planeación, plan educativo y demás relacionados con la formación por competencias. Para la recolección de datos se utilizó una encuesta semiestructurada tipo entrevista con profundidad media, con diecisiete (17) preguntas, donde se buscó identificar componentes reales de la educación superior en Colombia. Las respuestas dadas arrojaron también datos informativos, clasificatorios y comparativos.

La población tomada como referencia, fueron dieciocho (18) Instituciones de educación superior; cincuenta seis (56) entrevistados con diferentes roles dentro de las instituciones, tales como administrativos, docentes, estudiantes, egresados, de los cuales se obtuvo información relevante.

Las instituciones de educación superior públicas colombianas analizadas son la Universidad Nacional de Colombia, Universidad de Antioquia, el Servicio Nacional De Aprendizaje, Politécnico Jaime Isaza Cadavid, Institución Universitaria Pascual Bravo, Colegio Mayor de Antioquia e Institución Universitaria de Envigado.

Las instituciones de educación superior privadas encuestadas fueron la Universidad Pontificia Bolivariana, Universidad Centro de Estudios de la Salud CES y la Universidad Javeriana. 


\section{Revista científica, arbitrada e indizada, bajo la modalidad electrónica.}

\section{RESULTADOS}

El análisis de la información desde cada nivel educativo muestra un panorama diferenciador de las tendencias educativas o modelos pedagógicos implementados en las instituciones educativas a las cuales prestan sus servicios docentes, es una sólida base para la investigación puesto que el punto de vista, es variable según el nivel educativo, percepciones del aprendizaje, métodos educativo pueden influir de diferente manera pues la planeación pedagógica, la evaluación y técnicas didácticas aplicadas, se desarrolla de múltiples formas.

En este sentido, el análisis de los cargos entrevistados muestra mayor representatividad en los docentes, seguido por los egresados y áreas administrativas. Es importante resaltar que son los docentes los ejecutores del modelo educativo institucional, los cuales aportan tanto a la metodología educativa como a la tendencia pedagógica de la institución de educación superior hacia los estudiantes, al tener mejor y mayor representatividad es posible extraer metodologías aplicadas que son valiosas para el fin de este trabajo, los demás roles aportan valiosos conceptos desde su rol y percepción educativa.

El modelo pedagógico más representativo dentro de la población en las diferentes instituciones de educación analizadas, fue el constructivista, el cual es la base del modelo de formación por competencias, seguido por el humanista - social, que es usual en las instituciones de orden público. El modelo conductista, muestra una representación menor, este enfoque educativo pertenece la educación tradicional, instituciones privadas o religiosas en Colombia.

De igual forma, se evidencian algunas prácticas costumbristas, pero también, una evolución gradual, de inmersión - transición en los modelos constructivistas, humanistas, sociales que hacen parte de la formación basada en competencias.

El plan de estudios, malla curricular o diseño curricular que identifican los participantes en este estudio según las instituciones de educación superior analizadas, muestra que están enfocados en mayor proporción en la formación por competencias y construcción del conocimiento desde el hacer, ser y saber, lo cual es importante para la cualificación del egresado.

El ministerio de educación nacional (MEN) en Colombia, fomenta los planes de estudio y prácticas pedagógicas en el enfoque por competencias, el cual, dentro de su filosofía, busca 


\section{Revista científica, arbitrada e indizada, bajo la modalidad electrónica.}

generar impacto en la economía del país con sus egresados en las industrias, explora la maximización de los niveles de competitividad y productividad en los procesos empresariales, este análisis proporciona herramientas que fundamentan el objeto del presente trabajo, ya que se ve necesario tener un modelo claro para la implementación del planteado en las instituciones educativas. El análisis de la práctica docente es la más importante ya que una institución requiere de una planeación pedagógica exitosa, clara, definida en sus estudiantes, los datos muestran que, para los encuestados, las actividades relacionadas con el hacer y la práctica educativa son elementos básicos para la selección, así como organización de contenidos pedagógicos.

En el proceso de evaluación del docente al estudiante, se encuentran algunas debilidades, si se analiza desde la necesidad el modelo de formación por competencias, los datos muestran como el docente de educación superior enfatiza la evaluación, en los resultados o productos que entrega el estudiante durante la formación, cuando deberían prevalecer aspectos más competitivos desde su perfil de salida, desempeños prácticos, la constante evaluación de saber, hacer y ser en toda la integralidad del proceso educativo.

En el análisis del rol del docente en la educación superior de acuerdo con la información dada por los encuestados es magistral, es decir, expositivo en gran proporción, donde lo conceptual y lo impuesto como conocimiento prima, dicho rol, es base fundamental el modelo educativo tradicional.

En el análisis del rol del estudiante en las instituciones de educación superior teniendo en cuenta los resultados de las encuestas y entrevistas, se puede observar que los estudiantes son participativos, lo cual es valioso, por su aporte significativo en el proceso educativo, esto incita la integración en el proceso académico, en temas como prácticas, laboratorios, talleres, vivencias, visitas empresariales, reflejando la evolución tanto del docente, como de las instituciones de educación superior, incursionado en la diversificación de metodologías y técnicas en la enseñanza.

En el análisis generado por los aportes de los entrevistados, se reitera que el uso de las TIC, plataformas virtuales, medios tecnológicos, son componentes básicos en la educación superior. En Colombia no todas las instituciones de educación superior priorizan el uso de las plataformas virtuales como apoyo a la formación, pero con ayuda del Ministerio de Tecnologías de la Información y Comunicaciones, se han implementado estrategias y 
apoyos para que esto sea prevaleciente desde la educación básica primaria hasta la superior, aunque aún falta implementarlo por completo, un alto porcentaje de instituciones educativas que ya usan de plataformas virtuales de aprendizaje.

En el resultado de las encuestas y entrevistas aplicadas a las instituciones educativas, la interacción docente - estudiante, se evidencia que aún hay formadores con métodos pedagógicos excluyentes, magistrales, de poca relación con sus estudiantes; esto ya quedó en el pasado, el educador debe alinearse a los requerimientos de un mundo globalizado donde la educación es cambiante, participativa e incluyente. El análisis refleja docentes en búsqueda de estudiantes participativos, activos, interactuantes con el medio externo, investigadores, en buscadores de información, conocimiento, autoaprendizaje y mejoramiento continuo de sus competencias junto a sus desempeños.

El rol del estudiante cambió, pasa de ser un simple receptor de teorías y conocimientos, a un productor de aprendizajes significativos, apoyados de un docente instructor - asesor. El análisis muestra el uso de estrategias didácticas multi-activas en la educación superior. Finalmente, el docente, el egresado y estudiante, califican la didáctica como la mejor estrategia para impartir formación, una sola metodología ya no es suficiente para garantizar el conocimiento del estudiante de pregrado.

\section{REFLEXIONES FINALES}

Los resultados evidencian que la educación tradicional es predominante en las instituciones de educación superior en Colombia, tanto en lo administrativo como en lo académico. La planeación educativa está centrada en la administración de tiempos y recursos. Su gestión se encuentra desligada del sector productivo, por cuanto no existen convenios de cooperación que los relacionen permanentemente. El componente académico se centra en el desarrollo de programas con énfasis teórico-científico, en algunas ocasiones investigativo, aspectos importantes para el desarrollo del conocimiento y la ciencia, aunque insuficientes para superar la brecha entre los sectores educativo - productivo.

Se hace necesario llevar los procesos de gestión administrativa, de planeación - ejecución educativa desde los actuales estados formales pero un tanto estáticos de la educación tradicional a los más dinámicos, flexibles del modelo basado en competencias a la vez dar el salto desde los programas curriculares centrados en contenidos en la comprensión de los 
mismos del modelo tradicional, a los centrados en la formación por competencias de desempeño, la educación centrada en tal modelo, de modo que la generación de nuevos conocimientos y métodos tengan el propósito de innovar en el campo productivo.

Por último, se logra concluir, que para generar la transformación propuesta se han de identificar variables diferenciadoras tales como, el análisis de las necesidades en los sectores económicos, productivo, de servicios, los requerimientos de empleabilidad del país, necesidades empresariales e industriales, además de tener en cuenta las condiciones diferenciadoras para los niveles de formación como pregrado, postgrado, extensión, características de diferentes niveles de egresados (académicos, investigadores, productivos, creativos o innovadores), para dar respuesta a lo que requiere un país de los egresados y desde los currículos universitarios.

\section{REFERENCIAS BIBLIOGRÁFICAS}

Alvarez, C., Aguirre, G., \& Cifuenetes Davila, G. (2015). Nuevo Enfoque por Competencias. Domingo, 29 de Noviembre. Retrieved from http://nuevoenfoqueporcompetencias.blogspot.com/

Asún Inostroza, R., Zúñiga Rivas, C., \& Ayala Reyes, M. C. (2013). La formación por competencias y los estudiantes: confluencias y divergencias en la construcción del docente ideal. Calidad En La Educación, (38), 277-304. https://doi.org/10.4067/S0718-45652013000100008

Bolívar, A. (2011). Aprender a liderar líderes. Competencias para un liderazgo directivo que promueva el liderazgo docente. Educar, 47(2), 253-275. https://doi.org/10.13140/RG.2.1.4047.3126

Bortef, G. Le. (2001). Ingénierie et évaluation des compétences. (B. E. G. 2000, Ed.), Les Editionsd'Organization Le Boterf $G$. Construire les compétencesindividuelles et collectives ( $3^{\mathrm{a}}$ ed, Vol. 2). Barcelona.

Cabra Torres, F. (2008). La evaluación y el enfoque de competencias: Tensiones, limitaciones y oportunidades para la innovación docente en la universidad. Revista Escuela de Administración de Negocios, 2(63), 91-105. https://doi.org/10.21158/01208160.n63.2008.445

Cano, E. (2015). Como Mejorar las Competencias de los Docentes. Guia Para la Autoevaluacion Y el Desarrollo de Competencias del Profesorado. (G. D. I. SL, Ed.) (1Eedicion ed.). Barcelona: Cevagraf ESpaña. Retrieved from www.grao.com

Corado Fuentes, N. M. (2017). Texto Paralelo para el Curso E111.6 Diseño, Ejecución y Evaluación de Proyectos Curriculares Facultad de Humanidades, Universidad de San Carlos de Guatemala. Journal of Personality and Social Psychology, 1(1), 1188-1197. https://doi.org/10.1111/j.1469-7610.2010.02280.x

Díaz Barriga, Á. (2006). El enfoque de competencias en la educación. ¿ Una alternativa o 


\section{Revista científica, arbitrada e indizada, bajo la modalidad electrónica.}

un disfraz de cambio? Perfiles Educativos, XXVIII(111), 7-36. Retrieved from http://www.scielo.org.mx/pdf/peredu/v28n111/n111a2.pdf

Díaz Villa, M. (2002). F LEXIBILIDAD Y E DUCACIÓN S UPERIOR. (SECRETARÍA GENERAL - PROCESOS EDITORIALES., Ed.) (Primera Ed). Bogotá, D. C. Colombia: COPYRIGHT: ICFES 2002. Retrieved from file:///C:/Users/USER/OneDrive/maestria/FLEXIBILIDAD Y Y EDUCACIÓN SUPERIOR.pdf

Espinoza Cruz, M. (2009). LA AUDITORÍA PARA EL APROPIADO CONTROL INTERNO EN UNA INSTITUCIÓN EDUCATIVA DE NIVEL SUPERIOR. Tacna. Retrieved from http://www.upt.edu.pe/ouci/archivo/I - 254.2 web TI.pdf

García, J. (2011). Modelo Educativo Basado en Competencias: Importancia y Necesidad. Revista Electrónica “Actualidades Investigativas En Educación,” 11(3), 1-24.

García Retana, J. A. (2011). Modelo educativo basado en competencias: importancia y necesidad. Revista Electrónica “Actualidades Investigativas En Educación,” 11 (3), 1-24. https://doi.org/1409-4703

Graffe, G. J. (2002). Gestión educativa para la transformación de la escuela. Revista de $\begin{array}{llll}\text { Pedagogía, 23(68), } & \text { 495-517. } & \text { Retrieved }\end{array}$ http://www.scielo.org.ve/scielo.php?script=sci_arttext\&pid=S0798-

97922002000300007

Hernandez, R. (2014). Metodología de la investigación. Mc. Graw Hill Education. Mc Graw Hill Education. https://doi.org/- ISBN 978-92-75-32913-9

ITSA, I. U. (2018). Organismos de Control. Retrieved from http://itsa.edu.co/instituto/organismos-de-control

Leyton, A. E. G., \& Herrera, M. T. (2012). La formación de competencias de investigación a través de los métodos cualitativos en el análisis de la narrativa audiovisual. Comunicación: Revista Internacional de Comunicación Audiovisual, Publicidad y Estudios Culturales, 1(10), 1397-1408. Retrieved from https://dialnet.unirioja.es/servlet/articulo?codigo=3993351\%0Ahttps://dialnet.unirioja. es/servlet/citart?info=link\&codigo $=3993351 \&$ orden $=354935$

López Ortega, A. (2008). El Enfoque por Competencias en la Educación. In Universidad de Guadalajara (pp. 434-438). Guadalajara, Mexico.

Lozano Rosales, R. (2012). MODELO EDUCATIVO BASADO EN COMPETENCIAS EN UNIVERSIDADES POLITÉCNICAS EN MÉXICO: PERCEPCIÓN DE SU PERSONAL DOCENTE-ADMINISTRATIVO. Actualidades Investigativas En Educación, 12(2), 1-19. $\quad$ Retrieved from https://www.redalyc.org/comocitar.oa?id=44723437008

Marrero Sánchez, O., \& Lasso, M. del C. de la V. G. (2017). El proceso de enseñanzaaprendizaje por competencias. Una visión desde el enfoque sistémico. Congreso Universidad, 6(4), 28-46. Retrieved from file:///C:/Users/USER/Downloads/837-4820-1-10-20171026.pdf

Martínez, P., \& Echeverría, B. (2009). Formación basada en competencias. Revista de Investigación Educativa, 27(1), 125-147. Retrieved from http://revistas.um.es/rie/article/view/94331 
Ministerio de educación. (2011). Programa para la transformación de la calidad educativa. Guía Para Actores Involucrados En El Programa, 05, 75.

Ministerio de Educacion Guatemala. (2018). Las competencias y el currículo Fundamentos del Currículo. Retrieved from http://cnb.mineduc.gob.gt/wiki/Sección_6:_Las_competencias_y_el_currículo__Fundamentos_del_Currículo

MINISTERIO DE EDUCACION NACIONAL. (2016). articles343616_documento_sistema_aseguramiendo_calidad_ETyDH (1). Bogota.

Montemayor, L. E. (2011). PARA LA EDUCACIÓN SUPERIOR BASADA EN COMPETENCIAS. Revista de Investigación Educativa, 29, 205-218. Retrieved from file://C:/Users/USER/OneDrive/maestria/COMPETENCIAS QUE DEBE TENER UN DIRECTOR ACADÉMICO UNIVERSITARIO PARA LA EDUCACIÓN SUPERIOR BASADA EN COMPETENCIAS.pdf

Muro Lozada, Xiomara, \& Serrón Sergio, S. (2007). La agenda de investigación en el proceso de transformación de las instituciones de educación superior (IES). Paradígma, 28, 07-38. Retrieved from http://www.scielo.org.ve/scielo.php?script=sci_arttext\&pid=S101122512007000100002\&lng=es\&nrm=iso

Nacional., M. de E. (2018). Diseño y ajuste de programas bajo el enfoque de competencias. (M. de E. Nacional., Ed.), Revolucion Educativa Colombia Aprende. (Primera Ed). Bogotá, D. C. Colombia. Retrieved from https://www.mineducacion.gov.co/1759/articles-237704_Documento_6.pdf

Paredes, I., \& Inciarte, A. (2014). Modelo Pedagogico Formación Profesional Integral SENA, $1 \S$. Colombia.

Ramírez Montoya, M. S. (2013). Competencias Docentes y Prácticas Educativas Abiertas en Educación a Distancia. (E. LULU, Ed.) (1ra edició). México, DF: Ediciones LULU. $\quad$ Retrieved from https://books.google.com.co/books?hl=es\&lr=\&id=1_QVBgAAQBAJ\&oi=fnd\&pg=P A10\&dq=COMPETENCIAS+DOCENTES+Y+PRÁCTICAS+EDUCATIVAS+ABIE RTAS+pdf\&ots $=$ Nax04bqgy\&sig=zcaNQxkOPwkj0afM6_tYGwLngws\#v=onepage\&q

SENA. Proyecto Educativo Institucional SENA (2014).

Solar, M. I. (2005). EL CURRÍCULUM DE COMPETENCIAS EN LA EDUCACIÓN SUPERIOR : DESAFÍOS Y PROBLEMÁTICA. Pensamiento Educativo, 36(Junio), 172-191. Retrieved from file:///C:/Users/USER/Downloads/343-796-1-PB.pdf

Stabback, P. I. (2016). Qué hace a un currículo de calidad; Current and critical issues in the curriculum and learning; Vol.:2; 2016. UNESCO-IBE, 2, 40. Retrieved from http://unesdoc.unesco.org/images/0024/002439/243975s.pdf

UNESCO. (2017). Gestion educativa estrategica, IPPE.pdf (IIPE Bueno). Buenos Aires.

Vargas, J. M., Valencia, N., \& Vanegas, R. (2011). Una mirada de la formación por competencias para los procesos académicos de la facultad de ciencias militares de la Escuela Militar de Cadetes General José María Córdova *. Revista Educación y Desarrollo Social, 3(1), 21-36. 
Revista científica, arbitrada e indizada, bajo la modalidad electrónica.

Zapata, J. S. (2015). El modelo y enfoque de formación por competencias en la Educación Superior: apuntes sobre sus fortalezas y debilidades $1 \mathrm{~A}$ model and approach of competency-based training in higher education: strengths and weaknesses. Revista Academia y Virtualidad, 8(2), 24-33. 